Págs. 303-307

Tim Rudd and Ivor Goodson (ed.) (2017). Negotiating Neoliberalism. Developing Alternative Educational Visions. Rotterdam: Sense Publishers. ISBN: 978-94-6300-852-5, 219 páginas.

\title{
Adriana Marrero'
}

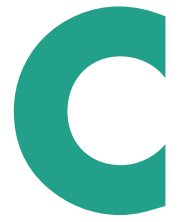

on frecuencia, la sociología de la educación se mueve con dificultad en el vasto campo que intermedia entre los estudios teóricos, muchas veces de inspiración filosófica, que permiten comprender y dar sentido a las grandes tendencias de los sistemas escolares en las sociedades contemporáneas, y la investigación empírica, por lo general, demasiado preocupada por la exactitud de la medición y la confiabilidad de los datos que pretenden representar aquellas tendencias. No es raro que así suceda. Por un lado, una acentuada conciencia teórica genera una visión más complejizante, más crítica, y menos pragmática frente a las necesidades de evaluación que enfrenta todo sistema educativo y suele, por ello afrontar directamente la tarea de discutir las bases, supuestos y consecuencias de esa evaluación. Por otro, la preocupación por la precisión de las evaluaciones, que ha sido parte importante de las discusiones sobre el estado de la educación en distintas partes del mundo en los últimos tiempos y de múltiples estudios comparativos, lleva implícito un cierto olvido de la «validez» sustantiva de la medición, esto es, un notorio silencio sobre la pregunta de qué es aquello que se está midiendo, cuál es su naturaleza y su historia, en qué tipo de relaciones está inscripto, y hasta qué punto, finalmente, esos indicadores realmente miden aquello que pretenden medir.

Lo teórico y lo empírico, el mundo de las ideas abstractas y de la experiencia, muchas veces cuantificada, suelen funcionar, en la sociología, como poderosos polos de atracción que no permiten ni la equidistancia, ni el diálogo. Una tercera vertiente, de origen etnográfico, elude la tensión entre esos dos polos, simplemente, prescindiendo de ambos. Sin teoría propiamente dicha, escudada en la ingenua pretensión de que es posible comprender la realidad sin supuestos teóricos previos, y evitando, también, pisar el complejo terreno de la recurrencia, que es el terreno de lo cuantitativo, pero también y sobre todo de lo social, se adentra en la perspectiva de unos sujetos que pocas veces son vistos, realmente, como actores y agentes inscriptos en una situación social concreta, de tiempo y de espacio. Las tres tradiciones y los tres modos de estudiar la educación y los sistemas escolares actuales, suelen convivir, o con extrema dificultad, o con recíproca indiferencia, configurando, en los hechos, tres comunidades separadas, con distintos puntos de partida, abordajes y métodos. Esto es particularmente cierto en el mundo hispano de los estudios sobre educación, pero es también un rasgo que caracteriza al trabajo sobre educación en muchas partes del mundo.

$1 \quad$ Universidad de la República (Uruguay), adriana.marrero.fernandez@gmail.com 
Tal vez sea por todo esto, que el libro que nos ofrecen Tim Rudd y Ivor Goodson, impacta al lector con un sorpresivo efecto de frescura, profundidad, agudo interés intelectual y, lo que más extraño, dado el tema sobre el que trata, mucha, muchísima motivación. Me refiero a «Negotiating Neoliberalism: Developing Alternative Educational Visions», publicado por Sense Publishers al comienzo en este mismo año 2017.

Las múltiples virtudes de este libro no son fáciles de sistematizar. Destacaré lo central: El neoliberalismo, en especial su impacto a partir de la crisis de 2007, como tema, como factor determinante de importantes cambios sociales y políticos, como factor distorsionante de las prácticas educativas y evaluativas de los docentes y en definitiva, de la vida de educadores y estudiantes afectados en práctica cotidiana y su proyección de futuro.

Cuando ya pensábamos que no quedaba nada nuevo para decir sobre el neoliberalismo y el modo cómo ha afectado las políticas públicas, los sistemas educativos, y las vidas de millones de docentes y estudiantes alrededor del mundo, el libro editado por Tim Rudd y Ivor Goodson, provee una visión más completa y más profunda del fenómeno, tal como se expresa en múltiples dimensiones, instituciones y actores. A diferencia de otros muchos trabajos, sin embargo, los autores logran, en un texto construido con notable maestría, evitar deslizarse hacia cualquiera de los dos extremos tan usuales en la literatura educativa hispanoamericana: o hacia el fatalismo y la impotencia frente a un fenómeno de alcances globales, por un lado, o por otro, hacia vagos y hasta cierto punto inconducentes llamados a una resistencia que queda sin conceptualización, ni análisis.

Desde el punto de vista teórico, el texto es de una riqueza y una densidad notables. Tras un estilo elegante y sofisticado, el lector atento percibirá una sólida estructura argumentativa que abreva de la teoría crítica y de los más recientes desarrollos teóricos en el campo de la sociología y la educación. El discurso vincula con facilidad las propiedades limitantes y recursivas de una estructura matrizada al impulso de la crisis y el empuje neoliberal, con las capacidades de agencia de los sujetos, tal como se expresan a la luz de sus propios modos de representarse su situación, y la relación entre su pasado y su forma de proyectarse hacia el futuro, a partir de sus propias narraciones autobiográficas. Según veremos luego, desde el propósito emancipatorio de su proyecto socioeducativo, los autores proponen herramientas teóricas, conceptuales y metodológicas a fin de generar procesos autorreflexivos de interpretación de la realidad para su transformación. Como se ve, entonces, no se trata solo de un aporte para una mejor interpretación de este mundo sumido en todas las consecuencias de las políticas de «austeridad» -las cuales son cuidadosamente problematizadas - sino de aportar en concreto, a todos los actores vinculados a la educación, un programa para la autocomprensión y modificación de las prácticas propias, con propósitos transformadores.

Estos puntos de partida teóricos y estos métodos de trabajo intelectual, sólidamente articulados a través de ejes claros de análisis, se reflejan a lo largo de los distintos capítulos del libro, que, a pesar de la diversidad de autores y de procedencias - distintos centros académicos de Inglaterra, Irlanda y Noruegalogra una consistencia y una unidad notables.

No es posible, en los límites de esta reseña, dar cuenta de las ideas fuerza de cada uno de los trece magníficos trabajos que componen este volumen. Por ello, me gustaría mostrar, al menos, dos de ellos. Por un lado, el que constituye el segundo capítulo del libro, «Aquí, allí y en todos lados: medición, evaluación y logro», de Michael Hayler, especialista en formación docente e integrante del Centro de Investigación Educativa de la Facultad de Educación de la Universidad de Brighton y parte del grupo de 
trabajo que lidera Ivor Goodson. Por otro, el trabajo de los propios editores de este libro, Ivor Goodson y Tim Rudd, en el capítulo de cierre, «Los límites de la educación neoliberal: refracción, reinterpretación y reimaginación».

El capítulo de Hayler, que centra su interés en los múltiples efectos de las mediciones estandarizadas de los aprendizajes en las prácticas y la vida de los educadores y estudiantes, así como en las dificultades para la evaluación tal como es entendida por los docentes, merecería formar parte del programa de lecturas obligatorias de todo investigador en educación. Hayler transita, con una destreza singular, entre políticas económicas y educativas derivadas del nuevo avance neoliberal, antecedentes teóricos y herramientas conceptuales, transcripciones de discursos y reflexiones de docentes a partir de un trabajo sistemático del autor de recolección de información autobiográfica, e interpretaciones del propio autor, que articulan y dan sentido a todos los anteriores. La objetividad de las reformas gubernamentales, la subjetividad de los actores, y el diálogo que entabla entre esas dimensiones el autor, resultan en un texto profundo, interesante, complejo e increíblemente atractivo.

De por sí, la evaluación, como sostiene Michael Hayler, es parte intrínseca del proceso educativo y no tiene por qué tener efectos negativos. En la medida en que se practique una evaluación formativa apoyada en la investigación, puede conducir, por el contrario, al empoderamiento de educadores y estudiantes, y motivarlos a la resistencia respecto a la nueva cultura de la rendición de cuentas. Y ello porque, a pesar de su carácter ideológico, esa cultura y la simple medición de resultados a la que va asociada, solo puede surtir efecto sobre los actores si llega a naturalizarse, y no va a naturalizarse mientras la evaluación siga estando asociada al mundo de lo valorativo, y no meramente, al de lo fáctico e instrumental.

Siguiendo el eje de análisis propuesto por Rudd y Goodson, Hayler da cuenta de las cinco «erres», que articulan los distintos trabajos: «Recuerdo», «Regresión», «Reconceptualización», «Refracción»y «Renovación», (Remembering, Regression, Reconceptualisation, Refraction and Renewal). Estos conceptos son entendidos como modos de resignificación de la situación, a efectos de repensar las posibilidades políticas de la educación, y ofrecer un «antídoto», al menos parcial, contra las reformas de la política educativa de los últimos treinta años, su retórica, su promoción y su normalización. La «Resistencia», que el autor propone como la sexta «R», está lejos de convertirse en una consigna vacía. Por el contrario, acá, la «resistencia» tiene como propósito reafirmar la escuela como un lugar desarrollo personal y progreso social. Concebidos como actores situados en la intersección entre la biografía, la sociedad y la historia, los docentes podrían constituirse como actores centrales de este proceso, en la medida en que se comprometan, interpreten y respondan a nivel micropolítico del aula, a través de interacciones significativas con niños y jóvenes. A través de un texto rico en reflexiones de docentes sobre su biografía y circunstancias, el autor fundamenta e ilustra la idea central de que, al otorgar sentido a sus propias vidas y a su trabajo, los actores individuales disponen de libertad para interpretar y cambiar su situación, al mismo tiempo que son constreñidos a ajustarse a ella. Y ello, porque el lugar de la resistencia se encuentra en la subjetividad misma, y constituye una lucha que se produce al mismo tiempo sobre aquello en lo que nos hemos convertido y en contra de aquello en lo que nos hemos convertido.

El libro, como decíamos, se cierra con un capítulo elaborado por los mismos editores, Ivor Goodson y Tim Rudd, «Los límites de la educación neoliberal: refracción, reinterpretación y reimaginación».

El texto desarrolla, de modo magistral, un andamiaje conceptual que se asienta en importantes antecedentes en la teoría sociológica más avanzada, a la que se incorporan nociones novedosas, profun- 
damente reveladoras y heurísticamente rendidoras para la interpretación, cuestionamiento y transformación de la realidad social y educativa. Por esto, el texto tiene, sobre todo, una clara intención de convertirse en un programa para la visualización y aprovechamiento de las oportunidades de agencia, en una realidad que podría aparecer, bajo otra óptica, como irresistible.

Goodson y Rudd centran su análisis en el concepto de «refracción», entendido como un instrumento teórico, y a la vez, metodológico, que permite entender el vínculo particular que se establece entre la influencia de la globalización neoliberal y las múltiples formas en que ésta es asimilada o rechazada por las diferentes realidades concretas. Desde el nivel «supra» o global, el neoliberalismo, se expresa de modos distintos a nivel macro o nacional, meso o institucional, y a nivel micro o de los actores individuales. $\mathrm{Al}$ igual que la luz se descompone y cambia su trayectoria al impactar contra distintas superficies, también, en el campo de lo social y educativo, la refracción abre posibilidades de emancipación, esto es, abre espacios que no quedan cooptados por las lógicas neoliberales. A los autores no solo le interesa describir el modo cómo esas influencias son refractadas, sino, además, conocer las causas y las motivaciones que generan esa refracción.

La construcción del concepto mismo de refracción, a partir de una estructura molar ordenada en un espacio de propiedades generado por dos ejes cartesianos, señala las tensiones y complementariedades entre la estructura y la acción, el pasado y los proyectos futuros de los actores, tal como aparecen objetivas en las narrativas biográficas de los sujetos. Cuanta mayor es la refracción, sostienen los autores, mejores son los resultados educativos observados en los distintos contextos nacionales. Forma también, parte del análisis el modo cómo se ha expresado, en diversos períodos, la relevante contradicción entre la independización de la lógica instrumental proveniente de los intereses del mercado, que afecta no solo a los procesos educativos, sino también a su evaluación, por un lado, y los procesos y propósitos de una educación pública basada en la solidaridad y en los valores colectivos, por otro.

Si la refracción significa oportunidades de agencia, la producción misma de la agencia pertenece al sujeto, en aquellos aspectos y lugares que no han sido colonizados y cooptados por la lógica instrumental del neoliberalismo. A partir de la puesta en valor de la capacidad reflexiva del ser humano frente a estos problemas, y del cuestionamiento del lugar de nuestra propia acción en ellos, es como puede generarse una política de vida que logre alinear, de modo cotidiano, cada una de las acciones con nuestros propósitos e ideales. Como se lee en una cita mencionada por los autores: «Supón que lograras esa revolución sobre la que hablas y con la que sueñas. Supón que tu bando haya ganado y tuvieras el tipo de sociedad que querías. ¿Cómo vivirías, personalmente, en esa sociedad? ¡Empieza ahora mismo a vivir asíl»

Goodson y Rudd se sitúan, en esto, muy lejos de las perspectivas teóricas que ven en los procesos modernos de exacerbación de la racionalidad instrumental y de pluralismo, el origen de la pérdida de sentido de la existencia. Bien al contrario, en una postura que, al mismo tiempo que deriva de una sólida conceptualización, muestra el lado más luminoso de ella, los autores ubican en el sentido vital, aquello que está incontaminado y es incorruptible por la lógica racional del mercado: no siempre pueden conquistar nuestras mentes, menos aún, nuestros corazones.

Tan riguroso como inspirador, el libro que ponen en nuestras manos Tim Rudd y Ivor Goodson es una oportunidad invalorable de volver a reflexionar, con cuidado, sobre todo aquello que hemos querido alguna vez como educadores, estudiosos y sociólogos de la educación; pero también como personas sensibles y reflexivas, en busca de un mundo mejor. Solo que esta vez, estamos invitados a lograrlo con 
la mejor guía posible: la de estos dos grandes intelectuales y colegas, que a través de estas páginas todos quienes se acerquen a ellas, los podrán sentir tan amigos, tan humanos y tan cercanos.

Tal vez por ello, han logrado también, reunir a un notable conjunto de distinguidos colegas y pensadores, también personas sensibles, que han contribuido con sus trabajos a este proyecto. Me limitaré a transcribir los nombres de estos autores y los capítulos con los que colaboran, en su orden y, por fidelidad, en idioma inglés, aunque sus aportes merecen la máxima atención como perspectivas y propuestas alternativas en educación, en el desafío al orden neoliberal que ha venido colonizando la educación. Esos capítulos, y sus autores, son:

1. Negotiating Neoliberal Education: A Positional Issue and an Oposicional Stance (Tim Rudd and Ivor Goodson); 2. Here, There and Everywhere: Measurement, Assessment and Attainment (Mike Hayler); 3. Against Academic Labour and the Dehumanisation of Educational Possibility (Richard Hall); 4. Neoliberal Schooling, Dehumanisation and an Education (Peter Humphreys); 5. Neoliberalism and the Value of Higher Education (Yvonne Downs); 6. Beyond 'Entrepreneurialism of the Self': What It Means to be a Student in the Neoliberal University (Nadia Edmond); 7. Refractions of the Global Educational Agenda: Educational Possibilities in an Ambiguous Policy Terrain (Ingunn Elisabeth Stray and Helen Eikeland Voreland); 8. Education Free for All: Outsourcing, Contracts and Conflicts of Interest in the UK (Deborah Philips); 9. Co-operative Education, Neoliberalism and Historical Perspectives: The Dilemmas of Building Alternatives (Tom Woodin); 10. Co-operative Education Against Neoliberalism (John Schostak); 11. Resisting Neoliberal Education: For Freedom's Sake (Stephen O'Brien); 12. There Is An-(No)-Other Way: Surfacing the Hidden Injuries of 'Austerity' - Resistance, Resilience and Professional Responsibility: The Irish Case (Ciaran Sugrue); 13. The Limits of Neoliberal Education: Refraction, Reinterpretation and Reimagination (Ivor F. Goodson and Tim Rudd).

En definitiva, el libro editado por Tim Rudd y Ivor Goodson, constituye una magnífica obra, un trabajo epistemológico, teórico y metodológico riguroso y consistente, y un aporte indispensable para repensar la educación, pero sobre todo, para comenzar a operar ya, de modo inmediato, sin más dilación, una nueva postura ante el mundo y la vida que, en la práctica cotidiana, nos permita aprovechar las múltiples oportunidades de agencia, que nos abre, como un crisol, la refracción. 